\title{
Author Index Vol. 68, 1995
}

Abiru,H. 33 Abrahamsen, T.G. 153 Achaval,M. 394 Adams, J.A. 342 Agata,Y. 404 Akino,T. 185 Akkoç,N. 100 Akyol,F. 100 Amato, M. 301 Amir, Y. 15 Aranda, J.V. 1 Arduini,D. 163 Aydin, A. 100

Bachofen,H. 301 Bancalari, E. 342 Baró,L. 55 Barry, P.W. 394 Bartolucci, M. 259 Bassotti,G. 259 Batenburg, J J. 128 Beharry, K.D.A. 1 Bel, F. van 91,200 Benders, M.J.N.L. 91 Bertotto,A. 259 Bessler,H. 15 Bestetti, G. 354 Beyaert,C. 87 Blum,J.W. 354 Bocchini, J.A., Jr. 10 Bocci,V. 104 Boehm, G. 19 Bor, M. van de 91 Bouillon, R. 377 Bowman, B.M. 368 Boza, J.J. 55 Bracci, R. 104 Bratlid,D. 246 Brune,T. 318 Buonocore, G. 104 Burri,P.H. 229,301

Cantarutti, F. 169 Capponi, A. 163 Carr,R. 264 Cashore, W.J. 135

Castellucci, G. 259 Catz, S.D. 141 Çevik,N.T. 100 Chatelain,A. 292 Cothran,D.L. Ill Coxam,V. 368 Crupi, S. 259 Cukierski, M. 62 Curstedt, T. 185 Curzi-Dascalova, L. 270 Cutrín,J.C. 141 Damke,B.M. 229 Dani,C. 169 Davis, A.T. 211 De Filippo, M. 104 Dehan,M. 270 DelivoriaPapadopoulos, M.

419 Deloof, S. 292 DelValle,J. 81 Delvin,E.E. 157 Djaldetti, M. 15 Dorrepaal, C.A. 91 Dousset, B. 87

Edwards, S.W. 264 El-Mohandes, A.A.E. 308 Escobar, C. 47

Fabietti, G.M. 259 Fairand, A. 221 Falconer, A.E. 264 Farstad,T. 246 Fernandez, M.d.C. 141

Feuer, W.I. 342 Forenza, N. 259 Forsythe, D.W. 325 Forsythe,I. 394 Fujii,Y. 254 Fujino, N. 404

Fukui, Y. 33

Gaultier,C. 270 Gazarian, M. 334 Gelhar,D. 81

Gerli,R. 259 Gil, A. 55 Gilhooly, J.T. 191 Gioia, D. 104 Goldberg, R.N. 342 Groenendaal, F. 419 Guadix, E.M. 55 Guajardo, L. 1 Guglielmi,A. 169 Guignard, J.-P. 175

Haaland, K. 75 Han,J.H. 404 Hansen, T.W.R. 135 Hart,J. 15 Hauser, B. 270 Hensel,D. 318

Hentschel,R. 318 Herrera, E. 282 Herting,E. 185 Hiraishi, S. 404 Hiramatsu, Y. 26 Hori, C. 254

Horiguchi, Y. 404 Husson, A. 221

Irken,G. 100 Ishii,Y. 254 Ishikawa, H. 412

Jain,L. 81 Jain,S.K. 10 Jorch,G. 318

Kahane,V.L. 141 Katona, I.M. 308 Kavukçu,S. 100 Kawamitsu, T. 254 Kent,G. 334 Kiang,E. 308 Klein, J. 334 Knudsen,A. 398 Konishi,Y. 254 Koren,G. 334 Kretchmer,N. 384 Krumbiegel, P. 19

429

Kudo,T. 26 Kuo,G. 342

Levy,E. 157 Levy, P. 81 Linden, J. Ill Llobera,M. 119 Lloyd, T.R. Ill López-Luna, P. 282 Luzzi,E. 104

McCulloch, K. 81 McGowan, J.E. 419 Marchal,F. 87 Martinez, O. 342 Martinez-Augustin, O. 55 Matherne, G.P. Ill Mattson, B. 62 Ménard,D. 157 Meneghetti, S. 169 Miller, M.A. 368 Miller, S.C. 368 Misawa, H. 404 Mishra,O.P. 419 Modanlou, H.D. 1 Mollnes, T.E. 153 Monin,P. 87 Muñoz,C. 282

Nakamura, H. 39 Nogami, H. 412 Nunez, M. 119 
O’Callaghan, C. 394 Offenbacher, S. 342 Oguchi, K. 404 Olgun,N. 100 Olsen,G.D. 191 Orderud,W.J. 75 Osiovich,H. 342 Özkan,H. 100

Padbury,J.F. 404 Pazzelli,P. 259 Peinado-Onsurbe, J. 119 Picciolini, E. 104 Pillers, D.-A.M. 191 Pinto, E. 175 Pittard,W.B. 325 Prahalada, S. 62

Qi,D. 368

Rabe,H. 318 Ramet,J. 270 Ransonet, L. 10 Renouf,S. 221 Rieder,M.J. 276 Rivas,R 1.308

Rizzo, G. 163 Robertson, B. 185 Roman, C. 200 Romanini, C. 163 Rudolph, A.M. 200

Salas,M. 47 Sato,Y. 26 Schäfer,K.P. 185 Schürch,S. 301 Serres,M.-A. 87 Setton,C.P. 141

Shah,M. 10 Shiota, K. 33 Silliman,K. 384 Sirota, L. 15 Smith, S.A. 191 Sola, A. 200 Soregaroli, M. 163 Spence, S. 62 Spinozzi, F. 259 Sterin-Speziale, N.B. 141 Straussberg, R. 15 Sudo,M. 254 Suguihara,C. 342 Sun,B. 185

Tachibana, T. 412 Taddio,A. 334 Takada,S. 39 Takeda,N. 404 Taylor, H. Ill Teichmann, B. 19 Thoresen, M. 75 Trevisanuto, D. 169 Tschanz, S.A. 229 Tsukahara, H. 254

Uetani, Y. 39

Vaccaro,R. 259 Vacher,P.-Y. 354 Vagliasindi, C. 259 Van Camp, G. 292 Vandvik,B. 153

VanHerck,E. 377 Verhaeghe, J. 377 Vetter,C. 62 Vidyasagar, D. 81 Vilaró,S. 119

Wagner, C.L. 325 Wahl,L.M. 308 Wall, M.A. 191 Wallenius,K. 426 Walther,FJ. 128 Wise,R. 10

Yamamoto, K. 33,254 Yamamoto, Y. 33 Yokoyama, N. 39

Zacchello,G. 169 Zanardo,V. 169

430

Author Index Vol. 68, 1995 